\title{
BMJ Open Protocol for a prospective, controlled study of assertive and timely reperfusion for patients with ST-segment elevation myocardial infarction in Tamil Nadu: the TN-STEMI programme
}

\author{
Thomas Alexander, ${ }^{1}$ Suma M Victor, ${ }^{2}$ Ajit S Mullasari, ${ }^{2}$ Ganesh Veerasekar, ${ }^{3}$ \\ Kala Subramaniam, ${ }^{4}$ Brahmajee K Nallamothu, ${ }^{5}$ for the TN-STEMI Programme \\ Investigators
}

To cite: Alexander T, Victor SM, Mullasari AS, et al. Protocol for a prospective, controlled study of assertive and timely reperfusion for patients with ST-segment elevation myocardial infarction in Tamil Nadu: the TN-STEMI programme. BMJ Open 2013:3:e003850. doi:10.1136/ bmjopen-2013-003850

- Prepublication history and additional material for this paper is available online. To view these files please visit the journal online (http://dx.doi.org/10.1136/ bmjopen-2013-003850).

Received 19 August 2013 Revised 23 October 2013 Accepted 23 October 2013

\section{CrossMark}

For numbered affiliations see end of article.

Correspondence to Dr Thomas Alexander; tomalex41@gmail.com

\section{ABSTRACT}

Introduction: Over the past two decades, India has witnessed a staggering increase in the incidence and mortality of ST-elevation myocardial infarction (STEMI). Indians have higher rates of STEMI and younger populations that suffer from it when compared with developed countries. Yet, the recommended reperfusion therapy with fibrinolysis and percutaneous coronary intervention is available only to a minority of patients. This gap in care is a result of financial barriers, limited healthcare infrastructure and poor knowledge and accessibility of acute medical services for a majority of its population.

Methods and analysis: This is a prospective, multicentre, 'pretest/post-test' quasi-experimental, community-based study. This programme will use a 'hub-and-spoke' model of an integrated healthcare network based on clusters of primary-care health clinics, small hospitals and large tertiary-care facilities. It is an 'all-comers' study which will enrol consecutive patients presenting with STEMI to the participating hospitals. The primary objectives of the study is to improve the use of reperfusion therapy and reduce the time from first medical contact to device or drug in STEMI patients; and to increase the rates of early invasive risk stratification with coronary angiography within 3-24 h of fibrinolytic therapy in eligible patients through changes in process of care. Outcomes will be measured with statistical comparison made before and after implementing the TN-STEMI programme. The estimated sample size is based on the Kovai Erode Pilot study, which provided an initial work on establishing this type of programme in South India. It will be adequately powered at $80 \%$ with a superiority margin of $10 \%$ if 36 patients are enrolled per cluster or 108 patients in three clusters. Thus, the enrolment period of 9 months will result in a sample size of 1500 patients.

Ethics: This study will be conducted in accordance with the ethical principles that have their origin in the current Declaration of Helsinki and 'ethical guidelines for biomedical research on human participants' as laid down by the Indian Council for Medical Research. All participating hospitals will still obtain local ethics committee approval of the study protocol and written informed consent will be obtained from all participants.

Dissemination and results: Our findings will be reported through scientific publications, research conferences and public policy venues aimed at state and local governments in India. If successful, this model can be extended to other areas of India as well as serve as a model of STEMI systems of care for lowincome and middle-income countries across the world. Registration: Trial is registered with Clinical trial registry of India, No: CTRI/2012/09/003002.

\section{INTRODUCTION}

In the last 40 years, cardiovascular disease in India has quadrupled, and the estimates suggest that, by 2020 , almost $60 \%$ of patients with cardiovascular disease worldwide will be Indian. ${ }^{1}$ One of the most ominous manifestations of cardiovascular disease is ST-elevation myocardial infarction (STEMI), which carries a grave prognosis if not treated promptly using reperfusion therapy to re-establish the flow in the occluded coronary artery. ${ }^{2}$ Unfortunately, national registry data from 89 cities suggest that Indian patients with STEMI frequently fail to receive an adequate reperfusion therapy and to a greater extent than comparable patients in the developed countries. ${ }^{3}$ For example, reperfusion therapy with fibrinolysis is received by less than $60 \%$ of Indian patients with STEMI and those that undergo it often do so after great delays. ${ }^{3}$ Furthermore, a few patients go on to early invasive evaluations and less than $10 \%$ receive 
percutaneous coronary intervention (PCI) during their hospitalisation despite growing support for this type of pharmacoinvasive approach. ${ }^{3}$ Improving access to these critical treatments is a key opportunity to improve STEMI care that has large implication for India as the epidemic of cardiovascular diseases continues to grow.

However, the challenges in improving STEMI care in India are formidable and include non-clinical factors, such as financial barriers, limited healthcare infrastructure and poor accessibility of acute medical services for a majority of its population. We therefore previously designed the Kovai Erode Pilot STEMI Study to assess the feasibility of developing a treatment model for STEMI in India based on analogous 'systems of care' developed in North America and Europe. ${ }^{4}$ This study was carried out in the rural district of Erode, located in the Northern part of Tamil Nadu. As a proof-of-concept study it demonstrated that by linking several smaller, peripheral 'spoke' hospitals with a centrally located, PCI-capable 'hub' hospital, the use and timelines of reperfusion therapy could be improved. Encouraged by these results, we now propose a broader 'hub-and-spoke' model in other areas of Tamil Nadu: the TN-STEMI programme. The purpose of this paper is to describe the framework and methods associated with this programme - the first-ever, multicentre study that aims to improve the delivery of reperfusion therapy in India. If successful, this programme can be extended to other areas of the country and serve as a model of STEMI systems of care for low-income and middle-income countries.

\section{METHODS AND ANALYSIS}

\section{Study design and objectives}

The TN-STEMI programme is a prospective, controlled, multicentre pretest/post-test quasi-experimental study that has been planned as a community-based treatment programme for improving the use and timeliness of reperfusion therapy in patients diagnosed with STEMI as confirmed by an ECG. Randomised controlled trial design was not chosen for this study, primarily because it was not attempting to prove the efficacy of a certain treatment or causality assessment. Instead, our goal was more focused on implementation of a programme that would facilitate a quick recognition and shifting of patients for definitive treatment following a standardised prehospital care, which are posed as challenges in STEMI care in India. It involves a stepwise approach that facilitates a rapid and definitive restoration of coronary blood flow using a combination of pharmacological and mechanical reperfusion therapies based on the presentation of the patient. This programme will use a 'hub-and-spoke' model that relies on an integrated healthcare network based on clusters of primary-care health clinics and small hospitals built around four large tertiary-care facilities that are capable of providing advanced cardiovascular services, including PCI and cardiac surgery. The primary objectives of the TN-STEMI programme is to use organised systems of care to: (1) improve the use of reperfusion therapy and reduce the time from first medical contact to device or drug in STEMI patients; and (2) increase the rates of early invasive risk stratification in eligible patients by employing a pharmacoinvasive strategy of reperfusion.

We plan to measure our ability to achieve this overall objective through explicit measurement of changes in processes of care before and after the introduction of the TN-STEMI programme. Secondary (and implicit) objectives include:

- Integrating care between emergency medical services (EMS) and acute-care clinics and hospitals at the community level in India, especially in rural areas.

- Providing a pragmatic model to understand the challenges associated with developing a national STEMI programme in India.

- To increase public awareness of appropriate STEMI care in India.

\section{Study population, facilities and enrolment period}

The TN-STEMI programme incorporates an inclusive, 'all-comers' study design. Consecutive patients aged 20 years or older with symptoms or signs consistent with acute coronary syndromes and ECG confirmation of STEMI will be enrolled. For entry into the study, an ECG must have evidence of myocardial injury showing $\geq 1-\mathrm{mm}$ ST-segment elevation in at least two anatomically contiguous limb leads (aVL to III, including-aVR), $\geq 1 \mathrm{~mm}$ ST-segment elevation in a precordial lead V4 through V6, $\geq 2 \mathrm{~mm}$ ST-segment elevation in V1 through V3 or a new left bundle branch block.

Both 'hub' hospitals and 'spoke' hospitals are included in each of the four clusters. The hub hospitals are four large tertiary-care hospitals with the capability for emergency cardiac catheterisation and PCI (see online supplementary table S1). The participating hub hospitals are divided into class A or B facilities depending on the availability of around-the-clock PCI at the hospital. The spoke hospitals have been selected based on their proximity to the hub hospitals. Spoke hospitals situated within $30 \mathrm{~min}$ reach to a hub hospital have been classified as class $\mathrm{C}$ while those beyond $30 \mathrm{~min}$ were class D hospitals. All participating units had to commit to complying with the study protocol and were required to be within the catchment area for available emergency ambulance services. Details of the hub-and-spoke model are discussed below.

Baseline data on management and outcomes of STEMI patients will be collected for 3 months from all the participating hospitals during an enrolment period that started in the fall of 2012. This data include patient's details with address and telephone number, demographic details, personal and medical history in detail such as smoking status, whether the patient is a current smoker or smoker in the past or non-smoker; if the patient is a smoker, then the details of quantity of consumption and duration will be collected. Clinical examination findings, 
investigations, diagnosis, treatment modality, medication details, cardiac catheterisation details and outcome will be noted. Baseline data will also include assessment of systems of care-how did the patient come to the hospital, total ischaemic time, the mode of treatment, transport, time intervals of onset of chest pain, time reached the hospital, time taken to perform ECG, time of starting of treatment, etc. The enrolment period will be 'rolling' for each of the hospitals and followed by 9 months of postimplementation data collection on STEMI patients after execution of the TN-STEMI programme. Outcomes that we will be evaluating are discussed in detail below and include data on processes of care that will be available during the hospitalisation and follow-up data for 1 year from the index event; the 1-year follow-up will either be a hospital visit, if it is the routine practice at the local site, or a telephonic follow-up if hospital visit is not required by the treating hospital. Estimated loss to follow-up is $20 \%$. This estimate is based on our experiences with clinical research in these communities, and is conservative.

\section{TN-STEMI programme: the hub-and-spoke model}

We have organised the network of hospitals within the TN-STEMI programme using a 'hub-and-spoke' model that recognises four classes of healthcare facilities that care for STEMI patients in India.

- Class A hospital: Class A hospitals are PCI-capable hospitals with healthcare teams available 24/7 for managing STEMI and its complications. Patients admitted to these hospitals typically undergo primary PCI with an aim of door-to-balloon time less than $90 \mathrm{~min}$.

- Class B hospital: These are PCI-capable hospitals, but primary PCI cannot be performed outside of working hours. Patients admitted to a class B hospital outside of working hours are typically treated with fibrinolysis with a goal of door-to-needle time less than $30 \mathrm{~min}$. Patients would be taken for catheterisation within the next 3-24 h, in the same hospital, and undergo PCI, if indicated. Patients at this class of hospitals may undergo primary PCI (like a class A hospital) if the patient arrives during working hours.

- Class C hospital-These are healthcare facilities and hospitals with the capability to perform and transmit ECGs and that are located within $30 \mathrm{~min}$ reach to a class A or B hospital. These hospitals do not have fibrinolysis capability. All class $\mathrm{C}$ hospitals would constitute the spokes of the class A/B hospitals. On confirmation of STEMI, the class $\mathrm{C}$ hospitals will activate Gunapati Venkata Krishna Emergency Management and Research Institute (GVK-EMRI) ambulance, transmit the ECG and transfer the patient to a class A/B hospital, depending on the availability of primary PCI. This process of transfer should ideally take less than $60 \mathrm{~min}$.

- Class D hospital-This class of healthcare facilities and hospitals have the capability to perform and transmit ECGs but are located beyond $30 \mathrm{~min}$ reach to a PCI-capable hospital. All of these are capable of providing fibrinolysis. All class D hospitals would constitute the spokes of class A/B hospitals. Patients arriving at a class D hospital are treated with fibrinolysis after confirmation of STEMI as per routine hospital practice. After informing the receiving hospital that is linked to it, transfer of the patient via GVK-EMRI ambulance for urgent catheterisation and, if indicated PCI, will be within the next 3-24 h.

Each class A/B hospital will be linked to approximately 3-15 referring class C/D hospitals. A full list of the participating hospitals organised by their classes is presented in online supplementary table S1. Figure 1 shows the 'hub-and-spoke' model; figure 2 shows a geographical map of Tamil Nadu showing the locations of clusters of 'hub-and-spoke' hospitals in the TN-STEMI programme.

\section{Key partners in TN-STEMI programme}

The TN-STEMI programme involves three key partners from both the public and private sectors.

1. Government of Tamil Nadu-Chief Minister's Health Insurance Scheme. A recent and important development that is relevant to the TN-STEMI programme in India has been the establishment and growth of government-sponsored social insurance coverage for healthcare among those below the poverty line. This has been a state-based development, including the Chief Minister's Health Insurance Scheme in Tamil Nadu. All the hub-and-spoke hospitals in each cluster will be covered by this programme. This requirement will ensure that patients from all social classes can receive timely and suitable treatment for STEMI with no out-of-pocket expenses incurred for their hospitalisation. The Chief Minister's Health Insurance scheme is currently operated by three insurance companies: Tiruvellore Thattai Krishnamachari (TTK) Healthcare, MediAssist and MD India Third Party Administrator.

2. EMRI Ambulance. GVK-EMRI operates as a public-private-partnership and is recognised as a not-for-profit entity. It is an organisation that has pioneered the development of EMS in India, including training the paramedics, technicians, nurses and physicians in emergency care. GVK-EMRI ambulance services may be activated by a patient with chest pain or a healthcare facility using the 'Call 108' system. Units within this EMS are capable of acquiring ECGs, transmitting ECGs to an 'on call cardiologist' for STEMI confirmation, locating the nearest PCI-capable hospital using global positioning system (GPS) provided by a coordinating centre, and transferring the patient to the closest hospital. GVK-EMRI ambulances will also transport patients from the spoke hospital to the hub hospitals after an initial evaluation and a possible treatment.

3. STEMI-India. STEMI-India is a not-for-profit entity dedicated to STEMI care in India led by physicians from across the country. The purpose of this 
Figure 1 Architecture of an ST-elevation myocardial infarction cluster.

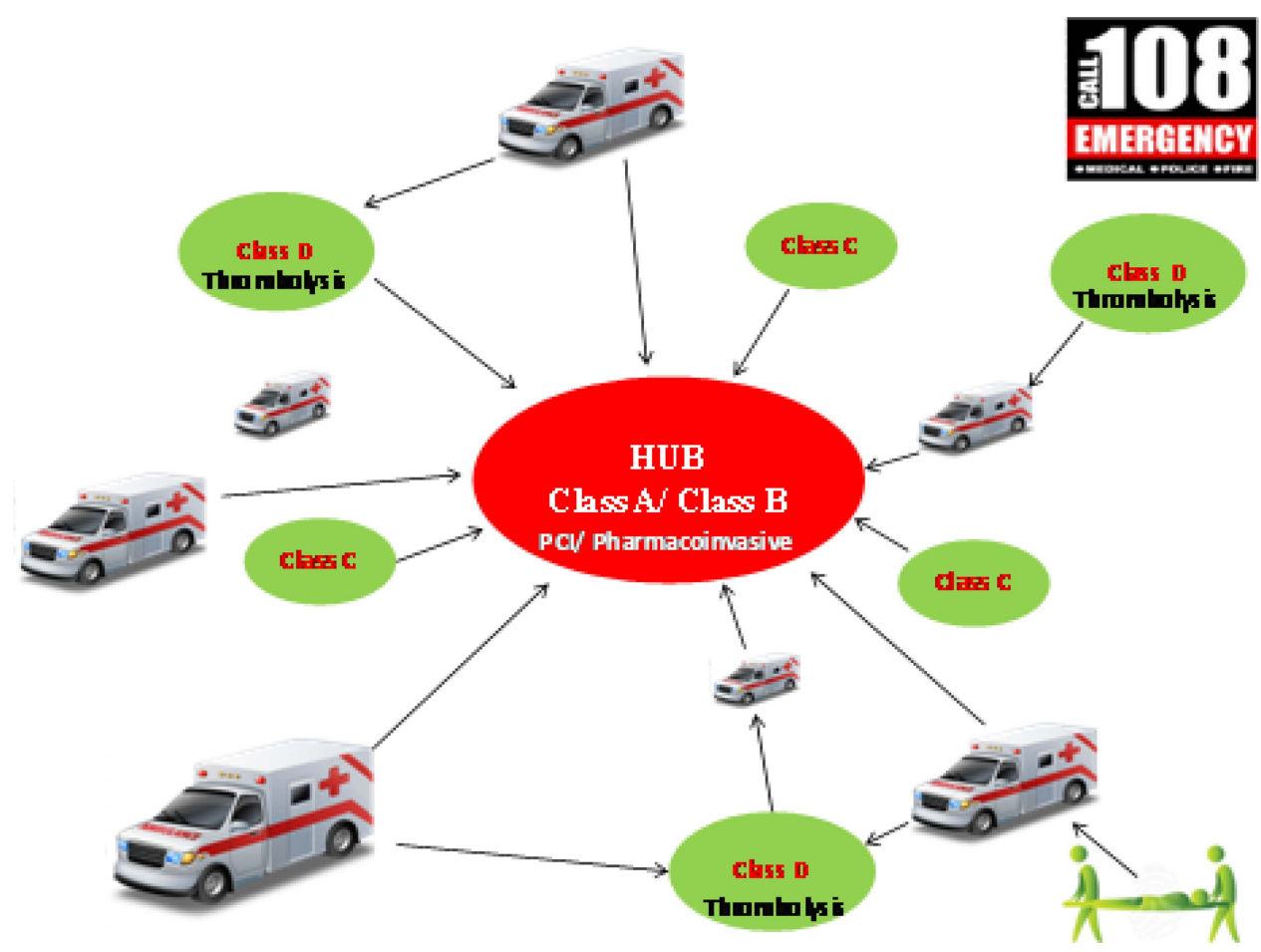

organisation is to review and disseminate the latest information from across the world on STEMI management to providers involved in STEMI care in
India; to help organise and train STEMI teams in hospitals; and to develop STEMI systems of care appropriate to the context of healthcare systems'

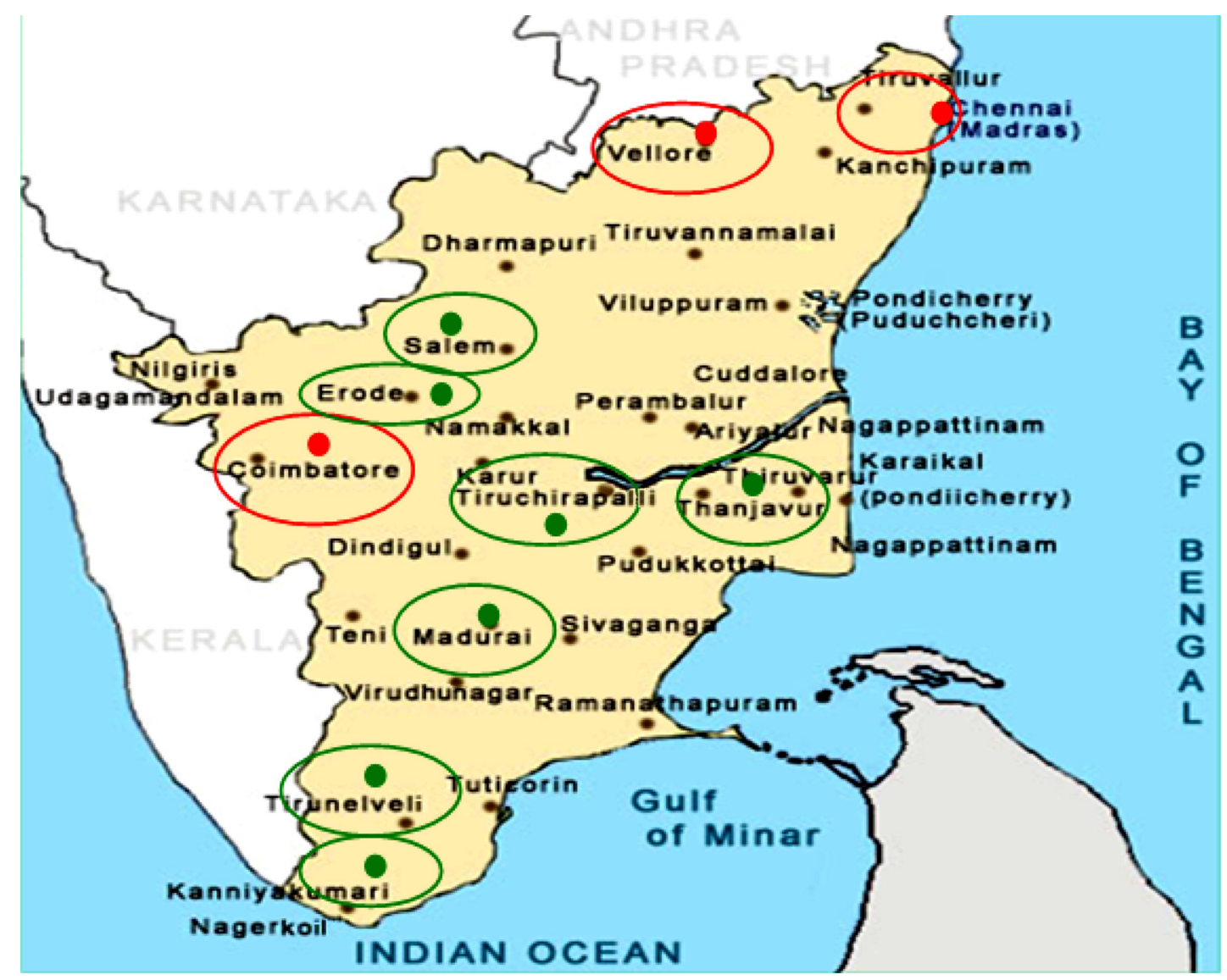

Figure 2 Geographical location of ST-elevation myocardial infarction clusters in the state of Tamil Nadu. 
needs and resources in India. Other goals include: facilitating and contributing to national STEMI guidelines within India and improving public education to reduce the delays in accessing care. The organisation's role in the TN-STEMI programme has been to provide expertise in the development and oversight of the study protocol to meet the standards of care within India for STEMI patients.

In addition to the three key partners above, we have partnered with two technology firms, Aosta and Maestro technologies, to coordinate the development of novel software and hardware (described below). Finally, Lotus Clinical Research Academy Pvt Ltd has been involved with the clinical administration of the trial. The TN-STEMI programme is supported by an independent grant from the Indian Council for Medical Research (ICMR).

\section{STEMII technology}

A novel aspect of the TN-STEMI programme is the implementation of new hardware and software components to optimise the performance and transmission of ECGs and other clinical information across the network of hospitals in India by paramedics, nurses and physicians. The hardware will comprise of the 'STEMI Kit' (figure 3) that includes:

1. ECG recording device-ECG devices will record the patient's ECG which would then be transmitted by the paramedics to a hand-held device of the STEMI coordinator in a hub hospital.

2. Vital signs monitoring device-This device will record the patient's vital signs and hemodynamic status, including pulse oxygen saturation, non-invasive blood pressure, heart rate and rhythm strip. This will also permit the transmission of key information by the paramedics to STEMI coordinators at hub hospitals.

3. Display and transmitting device-Each STEMI coordinator will have a hand-held device which collects and transmits data when required with sufficient battery support. This will allow for communication between STEMI coordinators and on-call cardiologists at the class $\mathrm{A} / \mathrm{B}$ hospitals.

Coordination of the hardware units by paramedics, STEMI coordinators and on-call cardiologists will be performed through unique software applications that are specifically designed for the TN-STEMI programme to be used across a universal platform for multiple devices. These are summarised in the online supplementary appendix.

The paramedic and STEMI coordinators will capture the patient demographic details along with a checklist for eligibility for fibronolytic therapy. This information along with the ECG will be transmitted to the on-call cardiologist in that cluster to diagnose STEMI and decide on initial treatment. The on-call cardiologist receives an alert once these data are obtained. She (or he) can then go over the patient records, ECG and confirm STEMI. This in turn alerts the paramedic to transport the patient to the destination based on GPS navigation. A dedicated server will be available round the clock to route all information from one hand-held device to the other in ambulance or hospital. Data are simultaneously stored on the server along with the ECG snapshot which can be accessed by teams at the receiving hospital.
Figure 3 ST-elevation myocardial infarction kit.

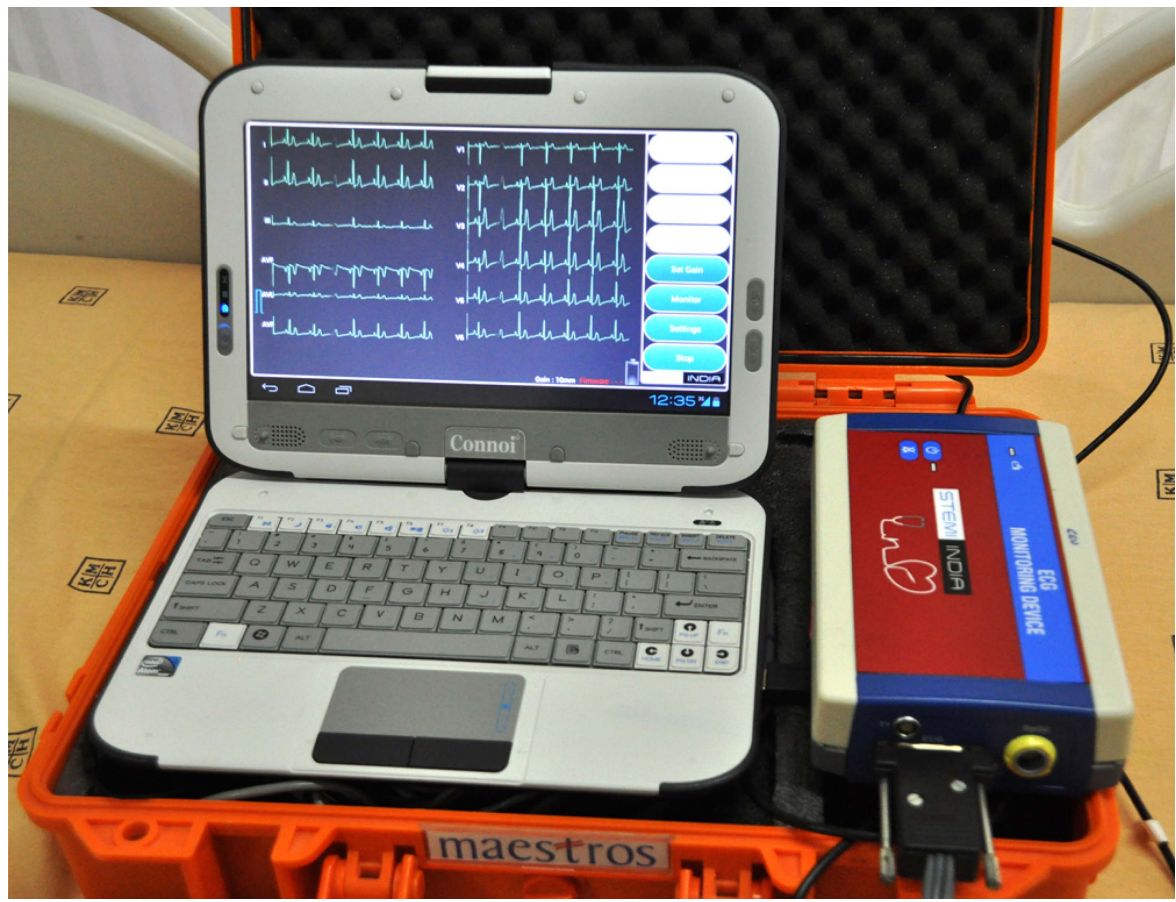




\section{Treatment protocols}

There will be two strategies to manage STEMI patients and both are adapted from current American College of Cardiology/American Heart Association and European Society of Cardiology guidelines for the context of the healthcare system of Tamil Nadu. ${ }^{5}$ Overall, these guidelines intend to minimise the total ischaemic time. ${ }^{7}$

1. Primary and rescue PCI: All patients with STEMI presenting to a class A hospital, class B hospital during working hours and class $\mathrm{C}$ hospital with an estimated transportation time of less than $60 \mathrm{~min}$ will undergo primary PCI. The aim is to achieve a door-to-balloon time of less than $90 \mathrm{~min}$ and a first medical contact to balloon time of less than $120 \mathrm{~min}$. Interhospital transfer of the patient from a spoke to a hub hospital is expedited from class D hospitals if: (A) fibrinolysis is contraindicated; or (B) unsuccessful fibrinolysis is clinically suspected with failed reperfusion (ie, rescue PCI).

2. Pharmacoinvasive strategy: All patients with STEMI presenting to a class B hospital outside of working hours or class D hospital with anticipated long transportation times, unless contraindicated, will receive fibrinolysis as per routine hospital practice with the goal of door-to-needle time less than $30 \mathrm{~min}$. The patients will then be transferred for an early invasive strategy with coronary angiography and PCI, if indicated, within 3-24 h of receiving fibrinolytic therapy.

All other medical therapy will be at the discretion of the treating physician and the healthcare team. However, the use of immediate dual antiplatelet therapy, in the form of aspirin and one of the thienopyridine group of drugs such as clopidogrel or prasugrel or ticagrelor and anticoagulation with heparin or equivalent drugs is encouraged. Other therapies, such as morphine, nitroglycerin, $\beta$-blockers and calcium-channel blockers, are not standardised.

All participating units will have a single protocol in place for reperfusion therapy and cardiac catheterisation laboratory activation. Prior to the implementation phase of the TN-STEMI programme, hospitals will adopt a specific strategy for reperfusion therapy based on distance, logistics, resources and equipment availability. STEMI kits consisting of 'Operations Manual and Guidelines for Management at First Medical Contact' will be circulated. These operations manual detail the interventional strategy at each point of care: the ambulance, the emergency department, interhospital transfer and the role of the hospitals. A STEMI coordinator responsible for accurate management of the project will be identified at each point of system care.

Patients with chest pain who call ' 108 ' will enter the system outside of the hospitals. These patients will be picked up by the EMRI ambulance, will have a preliminary ECG performed in the out-of-hospital setting, vital signs recorded and transmitted to the on-call cardiologist and STEMI coordinator. Once STEMI is confirmed by the on-call cardiologist, the ambulance will use GPS to locate the closest hospital in the cluster and reroute the patient there for appropriate treatment. Even before the patient reaches the hospital, the STEMI coordinator organises the cardiac catheterisation laboratory (if transport is to hub hospital) or coronary care unit for fibrinolysis (if transportation is to spoke hospital) based on the proximity of the patient to the nearest hospital. In the overall protocol for the TN-STEMI programme, any patient with cardiogenic shock will be taken directly to a class A/B hospital for primary PCI, bypassing a referring hospital even if it is the closest hospital.

\section{Primary and secondary outcomes}

Our primary outcomes will be based on care provided during the hospitalisation and process measures associated with the use of and time to reperfusion therapy. The secondary outcomes will be clinical events that occur during the hospitalisation and follow-up, although we recognise that we will be underpowered to detect differences in these events. These are detailed below. The reference group for comparison of the outcomes in this study will be the preprogramme implementation group.

\section{Primary outcomes}

- Use of reperfusion therapy with either fibrinolytic therapy or primary PCI.

- Use of timely reperfusion defined as door-to-balloon time $\leq 90 \mathrm{~min}$ or door-to-needle $\leq 30 \mathrm{~min}$ in patients with STEMI treated with primary PCI and fibrinolytic therapy, respectively.

- Use of early invasive risk stratification with coronary angiography and PCI in patients treated with fibrinolytic therapy.

\section{Secondary outcomes}

- Use of rescue PCI in patients with failed fibrinolysis.

- Composite of the following in-hospital outcomes: mortality, recurrent infarction/ischaemia, stroke, major and minor bleeds.

- Composite of the following outcomes at 1 year: allcause mortality, cardiac mortality, stroke, recurrent infarction/ischaemia, major and minor bleeds.

- Use of evidence-based therapies-aspirin, $\beta$-blocker and statin-on admission, at discharge and during follow-up.

\section{Statistical considerations}

Data from all centres will be combined for analysis. Data will be collected and processed into a quality assured database. Descriptive data will be provided in statistical summary tables and listings. Graphical presentations may also be presented where necessary. Continuous variables will be summarised using descriptive statistics such as mean, SD, coefficient of variation (\%), median, minimum and maximum; and the same will be reported. For categorical data, the number and percentage of participants in each category will be reported, along with $95 \%$ two-sided CIs where appropriate. Comparison between patients treated during the 
enrolment period and postimplementation periods based on the study outcomes will be tested using applicable test of hypothesis such as t test, $\chi^{2}$ test or nonparametric tests.

Eligible patients from the study with reference to the definition of control population and intervention population will be used for statistical comparison. Control population is defined as population included prior to implementation of TN-STEMI programme into a cluster that will provide baseline data for evaluation of operational parameters and outcomes before and after the intervention (ie, the enrolment period). Intervention population as per protocol is defined as patients presenting to hub-and-spoke centres after initiation of a cluster into TN-STEMI programme (ie, the postimplementation period). This will also include patients who were transferred and completed the study protocol and patients who were transferred and did not complete the study prior to intervention and postintervention.

The estimated sample size for the present study that allows for statistical inference for the primary end point uses the Kovai Erode pilot study as reference. ${ }^{4}$ It is estimated that the study would be adequately powered at $80 \%$ with a superiority margin of $10 \%$ if 36 patients are enrolled per cluster or 108 patients in three clusters. Considering the design of the study (all comers) and the objective of a state-wide programme implementation, the enrolment period of 9 months will result in a sample size of 1500 patients. If the sample size cannot be fulfilled during the study time frame, the enrolment period will be extended.

\section{Study oversight, ethical considerations and data collection and quality}

This study will be conducted in accordance with the ethical principles that have their origin in the current declaration of Helsinki and 'ethical guidelines for biomedical research on human participants' as laid down by the ICMR. Despite the fact that this is an implementation study focusing on quality improvement (QI), all participating hospitals will still obtain local ethics committee approval of the study protocol and written informed consent will be obtained from all participants. The rights, safety and well-being of the study participants are the most important considerations and should prevail over interests of society and science.

\section{Data collection and quality control}

Data will be collected prospectively from all the participating units from personnel blinded to the aims of the study. Electronic case report forms (eCRFs) must be completed for each patient screened/enrolled and the data for this study will be collected with an electronic data capture application. As required by the ICH GCP (International Conference on Harmonisation Good Clinical Practice) guidelines and regulatory authorities, the investigator will allow direct access to all pertinent medical records in order to allow the verification of data gathered in the eCRFs and for the review of the data collection process. Data will be captured and processed into a quality assured database. The investigator(s)/institution(s) will permit study-related monitoring, audits and regulatory inspection(s), providing direct access to source data documents. The periodic quality check will be performed to ensure the proper functioning and coordination of TN-STEMI network (hub-and-spoke model), timely transmission to class A/Class B hospitals and to minimise the treatment delays. Periodic QI reports will be generated for every participating hub hospital to ensure that quality systems are in place. QI will include a review of system administration/organisational activities, prehospital and hospital care. It will also have a documentation of effectiveness of hospitals and EMS service. A Data and Device Safety Monitoring Board (DDSMB) will periodically review and evaluate the accumulated study data for participant safety, study conduct, study progress, and make recommendations concerning the continuation, modification or termination of the project.

\section{DISCUSSION}

Reperfusion therapy is critical in the management of patients with STEMI and one of the most powerful predictors of early and late survival; however, its use is considerably hampered by several non-clinical and system-related barriers in low-to-middle income countries like India. Furthermore, primary PCI has increasingly become the preferred method of reperfusion therapy in STEMI management but because of the additional resources that it requires it is frequently unavailable in these settings. Expanding population-wide availability of reperfusion therapy, primary PCI and early invasive risk stratification are critical aspects of STEMI systems of care that have been used with great success in Western Europe and North America.

We believe that the TN-STEMI programme will create new opportunities to deliver these therapies in India by addressing various clinical, logistical and societal factors. As in other countries, effective management of STEMI at the community level in India will require executing proven treatment protocols along with efficient and rapid interhospital transfer within coordinated hospital networks. Regional systems of care such as 'Mission: Lifeline' have been successfully used in STEMI management in the USA. ${ }^{8}$ In Europe, 'hub-and-spoke' model of STEMI networks also demonstrate an improved adherence to reperfusion therapy and timely treatment strategies $^{9}$ and are gaining endorsement through the Stent-For-Life programme of the European Society of Cardiology. Although STEMI management in India may also benefit from such organised systems of care, there are little or no data to support that these approaches improve the key processes of care or outcomes in this 
environment. Hence, understanding the effect of the STEMI network implemented in the state of Tamil Nadu will have substantial implications for the country. This approach is particularly worthwhile as it leverages unique public and private partnerships, technological innovation in monitoring devices, an expanding EMRI ambulance system and novel strategies for reperfusion therapy and early invasive risk stratification. If successful, this type of network may be extended to the rest of India and even worldwide.

\section{Author affiliations}

${ }^{1}$ Department of Interventional Cardiology, Kovai Medical Center and Hospital, Coimbatore, Tamil Nadu, India

${ }^{2}$ Department of Interventional Cardiology, Madras Medical Mission Hospital, Chennai, Tamil Nadu, India

${ }^{3}$ Department of Epidemiology, Kovai Medical Center and Hospital, Coimbatore, Tamil Nadu, India

${ }^{4}$ Clinical Research, Lotus Clinical Research Academy Pvt. Ltd, Chennai, Tamil Nadu, India

${ }^{5}$ University of Michigan, Ann Arbor, Michigan, USA

Contributors TA and ASM participated in conception of trial design and provided the important intellectual content; SMV participated in drafting the article; GV participated in drafting tables and images; KS provided the statistical design and BKN participated in final correction and approval of the version.

Funding The study is funded by STEMI-India, Indian Council of Medical Research (ICMR). This study has been accepted by Indian Council of Medical Research and is still awaiting release of funds.

Competing interests None.

Ethics approval Ethical Committee in each hub hospitals.

Provenance and peer review Not commissioned; externally peer reviewed.
Data sharing statement This study is a protocol and detailed work book regarding the same is available with STEMI-India and LCRA.

Open Access This is an Open Access article distributed in accordance with the Creative Commons Attribution Non Commercial (CC BY-NC 3.0) license, which permits others to distribute, remix, adapt, build upon this work noncommercially, and license their derivative works on different terms, provided the original work is properly cited and the use is non-commercial. See: http:// creativecommons.org/licenses/by-nc/3.0/

\section{REFERENCES}

1. Kohn D. Getting to the heart of the matter in India. Lancet 2008;372:523-4.

2. American College of Emergency Physicians; Society for Cardiovascular Angiography and nterventionsO'Gara PT, Kushner FGAscheim DD, et al. 2013 ACCF/AHA guideline for the management of ST-elevation myocardial infarction: a report of the American College of Cardiology Foundation/American Heart Association Task Force on Practice Guidelines. J Am Coll Cardiol 2013;61:e78-140.

3. Xavier D, Pais P, Devereaux PJ, et al. CREATE registry investigators. Treatment and outcomes of acute coronary syndromes in India (CREATE): a prospective analysis of registry data. Lancet 2008:371:1435-42.

4. Alexander T, Mehta S, Mullasari A, et al. Systems of care for ST-elevation myocardial infarction in India. Heart 2012;98:15-17.

5. Silber S. Evidence based management of ST-segment elevation myocardial infarction (STEMI) latest guidelines of the European society of cardiology (ESC), 2010. Herz 2010;35:558-64.

6. Antman EM, Anbe DT, Armstrong PW, et al. ACC/AHA guidelines for the management of patients with ST-elevation myocardial infarction -executive summary. Circulation 2004:110:588-636.

7. Denktas EA, Anderson $\mathrm{VH}, \mathrm{McC}$ arthy $\mathrm{J}$, et al. Total ischemic time: the correct focus of attention for optimal ST-segment elevation myocardial infarction care. J Am Coll Cardiol Intv 2011;4;599-604.

8. Diercks D. American Heart Association Mission Lifeline: developing a STEMI, Regional Care System. http://www.emcreg.org/publications/ monographs/acep/2009/ACEP2009_dbd.pdf (accessed 30 Jul 2013).

9. Claeys MJ, Sinnaeve PR, Convens C, et al. STEMI mortality in community hospitals versus $\mathrm{PCl}$-capable hospitals: results from a nationwide STEMI network programme. Eur Heart J 2012;1:40-7. 